\title{
Discovery of the combined oxidative cleavage of plant xylan and cellulose by a new fungal polysaccharide monooxygenase
}

\author{
Matthias Frommhagen ${ }^{1}$, Stefano Sforza ${ }^{1,2}$, Adrie H Westphal ${ }^{3}$, Jaap Visser ${ }^{4}$, Sandra W A Hinz ${ }^{4}$, \\ Martijn J Koetsier ${ }^{4}$, Willem J H van Berkel ${ }^{3}$, Harry Gruppen ${ }^{1}$ and Mirjam A Kabel ${ }^{1 *}$
}

\begin{abstract}
Background: Many agricultural and industrial food by-products are rich in cellulose and xylan. Their enzymatic degradation into monosaccharides is seen as a basis for the production of biofuels and bio-based chemicals. Lytic polysaccharide monooxygenases (LPMOs) constitute a group of recently discovered enzymes, classified as the auxiliary activity subgroups AA9, AA10, AA11 and AA13 in the CAZy database. LPMOs cleave cellulose, chitin, starch and $\beta$-( $1 \rightarrow 4$ )-linked substituted and non-substituted glucosyl units of hemicellulose under formation of oxidized gluco-oligosaccharides.

Results: Here, we demonstrate a new LPMO, obtained from Myceliophthora thermophila C1 (MtLPMO9A). This enzyme cleaves $\beta$ - $(1 \rightarrow 4)$-xylosyl bonds in xylan under formation of oxidized xylo-oligosaccharides, while it simultaneously cleaves $\beta$ - $(1 \rightarrow 4)$-glucosyl bonds in cellulose under formation of oxidized gluco-oligosaccharides. In particular, MtLPMO9A benefits from the strong interaction between low substituted linear xylan and cellulose. MtLPMO9A shows a strong synergistic effect with endoglucanase I (EGI) with a 16-fold higher release of detected oligosaccharides, compared to the oligosaccharides release of MtLPMO9A and EGI alone.

Conclusion: Now, for the first time, we demonstrate the activity of a lytic polysaccharide monooxygenase (MtLPMO9A) that shows oxidative cleavage of xylan in addition to cellulose. The ability of MtLPMO9A to cleave these rigid regions provides a new paradigm in the understanding of the degradation of xylan-coated cellulose. In addition, MtLPMO9A acts in strong synergism with endoglucanase I. The mode of action of MtLPMO9A is considered to be important for loosening the rigid xylan-cellulose polysaccharide matrix in plant biomass, enabling increased accessibility to the matrix for hydrolytic enzymes. This discovery provides new insights into how to boost plant biomass degradation by enzyme cocktails for biorefinery applications.
\end{abstract}

Keywords: Biorefinery, LPMO, Cellulose, Endoglucanase, Myceliophthora thermophila C1, Xylan

\section{Background}

Effective degradation of plant polysaccharide biomass into monosaccharides, which are useful building blocks for biochemicals or biofuels, requires a variety of enzymes. Commercial enzyme cocktails for plant cell wall degradation mostly comprise enzymes produced by filamentous fungi, such as Aspergillus and Trichoderma

\footnotetext{
*Correspondence: mirjam.kabel@wur.nl

1 Laboratory of Food Chemistry, Wageningen University, Bornse

Weilanden 9, 6708 WG Wageningen, The Netherlands

Full list of author information is available at the end of the article
}

strains. In addition, the commercially available fungus Myceliophthora thermophila $\mathrm{C} 1$ is a good candidate for the production of thermotolerant carbohydrate-active enzymes [1]. Still, improvements of already existing enzyme cocktails are required for a more efficient degradation of plant biomass and a decrease in costs in the production of biochemicals and biofuels.

In general, plant cell walls are composed of a primary and secondary layer, which are both built from various polymers such as polysaccharides, lignins and proteins. The polysaccharides of the plant cell wall comprise 
cellulose fibrils and hemicellulose [2-5]. Cellulose is a homogeneous linear polymer of $\beta$ - $(1 \rightarrow 4)$-linked glucosyl units and, depending on the source, exceeding a degree of polymerization (DP) over 10.000 [6]. These $\beta$-(1 $\rightarrow 4)$-linked glucosyl chains form microfibrills via hydrogen bonds and van der Waals forces [7]. Depending on these interactions, cellulose is composed of crystalline and amorphous regions [8]. Lignin is an aromatic heteropolymer consisting of the three monolignols, coniferyl, sinapyl and p-coumaryl alcohol, which are methoxylated in various degrees. The network of lignin in the plant cell wall is built up of ester and ether linkages with hemicellulose $[3,9,10]$. Hemicellulose is, unlike cellulose, a very heterogeneous polymer. The hemicellulose structure differs between species of mono- and dicotyls and, depending on the source, can consist of a xylan, mannan, xyloglucan or $\beta$-glucan backbone [11-16]. The majority of these backbones are composed of $\beta-(1 \rightarrow 3$, $1 \rightarrow 4)$-linked xylosyl, $\beta$-( $\rightarrow$ 4)-linked mannosyl and $\beta$-(1 $\rightarrow 3,1 \rightarrow 4)$-linked glucosyl residues, respectively. In addition, these backbones show structural side-chain variations, which can differ in, i.e. type of substituent or distribution along the backbone [17]. Such hemicelluloses are strongly associated with cellulose via hydrogen bonding, especially the ones with a low degree of substitution or a block-wise distribution of substituents along the backbone [2, 4]. The cellulose-associated hemicelluloses block cellulases from reaching their substrate, which is likely to contribute to the defense of plants against microbial attack, and hinder the deconstruction of cellulose by industrial enzymes into fermentable monosaccharides $[18,19]$. Hence, degradation of cellulose-associated hemicelluloses is essential to improve the hydrolysis of cellulose present in the plant biomass.

It has been known for a long time that enzymatic degradation of cellulosic plant biomass can be achieved by a variety of glycoside hydrolases (GHs; EC.3.2.1.), which are all listed in the Carbohydrate-Active enZYmes (CAZy, [20]) database. However, the effectiveness of hydrolases on cellulose is limited, due to hydrogen bonds between the glucan chains of the rather crystalline cellulose. Recently, it was demonstrated that the breakdown of crystalline polysaccharides could be boosted by the action of lytic polysaccharide monooxygenases (LPMOs). These LPMOs are classified as subgroups, AA9, AA10, AA11 and AA13, in the CAZy database [21]. The LPMOs exhibit oxidative cleavage between $\beta$ - $(1 \rightarrow 4)$-glucosyl units in chitin, cellulose, hemicellulosic glucan and soluble cellodextrins under formation of oxidized gluco-oligosaccharides [22-26]. Depending on the type of LPMO, the products released are gluco-oligosaccharides that are oxidized at either the reducing $(\mathrm{C} 1)$ or the non-reducing (C4) end. Although not relevant for degradation of plant cell walls, very recently starch-active LPMO has been reported, cleaving between $\alpha-(1 \rightarrow 4)$-glucosyl units in starch under formation of C1-oxidized dextrins [26, 27]. This finding illustrates that LPMO activities cover a broader range of substrates than that earlier conceived. So far, no LPMO active on xylans has been reported.

In the present research, a new enzyme LPMO activity is described, which can be considered to be highly important for the concerted degradation of plant cellulose associated with xylan. In brief, we propose that the thermotolerant enzyme MtLPMO9A from $M$. thermophila $\mathrm{C} 1$ cleaves cellulose-associated xylan under formation of oxidized xylo-oligosaccharides. In addition, to evaluate the enhanced cellulose accessibility, the synergistic effect of $M t$ LPMO9A with an endoglucanase I was determined.

\section{Results}

\section{Enzyme purity}

From the $M$. thermophila $\mathrm{C} 1$ genome, protein $M t \mathrm{LP}-$ MO9A was predicted to be an LPMO belonging to subfamily AA9 [21]. Since the addition of LPMOs to a cellulase cocktail was found to considerably increase the release of glucose from cellulose $[28,29]$, it was of interest to analyze the mode of action of $M t$ LPMO9A in further detail. Hereto, $M t$ LPMO9A was expressed and produced in a protease/(hemi-) cellulase-free $M$. thermophila $\mathrm{C} 1$ strain with Dyadic technology (Dyadic NL, Wageningen, The Netherlands). $M t$ LPMO9A was purified to apparent homogeneity using multiple chromatographic steps. The purified enzyme showed a single band in SDS-PAGE with an apparent molecular mass of $23 \pm 1 \mathrm{kDa}$ (Additional file 1), in good agreement with the predicted mass of MtLPMO9A (22,755.3 Da; without signal peptide).

To further analyze the purified $M t$ LPMO9A preparation, the enzyme was subjected to LC/UV/ESI mass spectrometry. The elution pattern (Figure 1) showed a main and a shallow peak, of which the latter was due to protein denaturation by the LC conditions applied. MS analysis of both the main and shallow peak showed exactly the same protein mass based on the multi-charged ion patterns corresponding to a mass of 22,765.3 $\pm 0.1 \mathrm{Da}$. The $M t$ LPMO9A protein comprised $\pm 99.5 \%(65+34.5 \%$, from the main and shallow peak, respectively) of the total protein present based on UV $(214 \mathrm{~nm})$, and $94.7 \%$ based on total ion current (TIC) in the full mass range. In conclusion, $M t$ LPMO9A was obtained in an extremely pure form.

\section{Structural model of MtLPMO9A}

A structural model of $M t$ LPMO9A was generated based on the available structure of TtPMO1 from Thielavia terrestris [29] (Protein Data Bank entry: 3eii), which shared $75 \%$ amino acid sequence identity. The $M t$ LPMO9A 


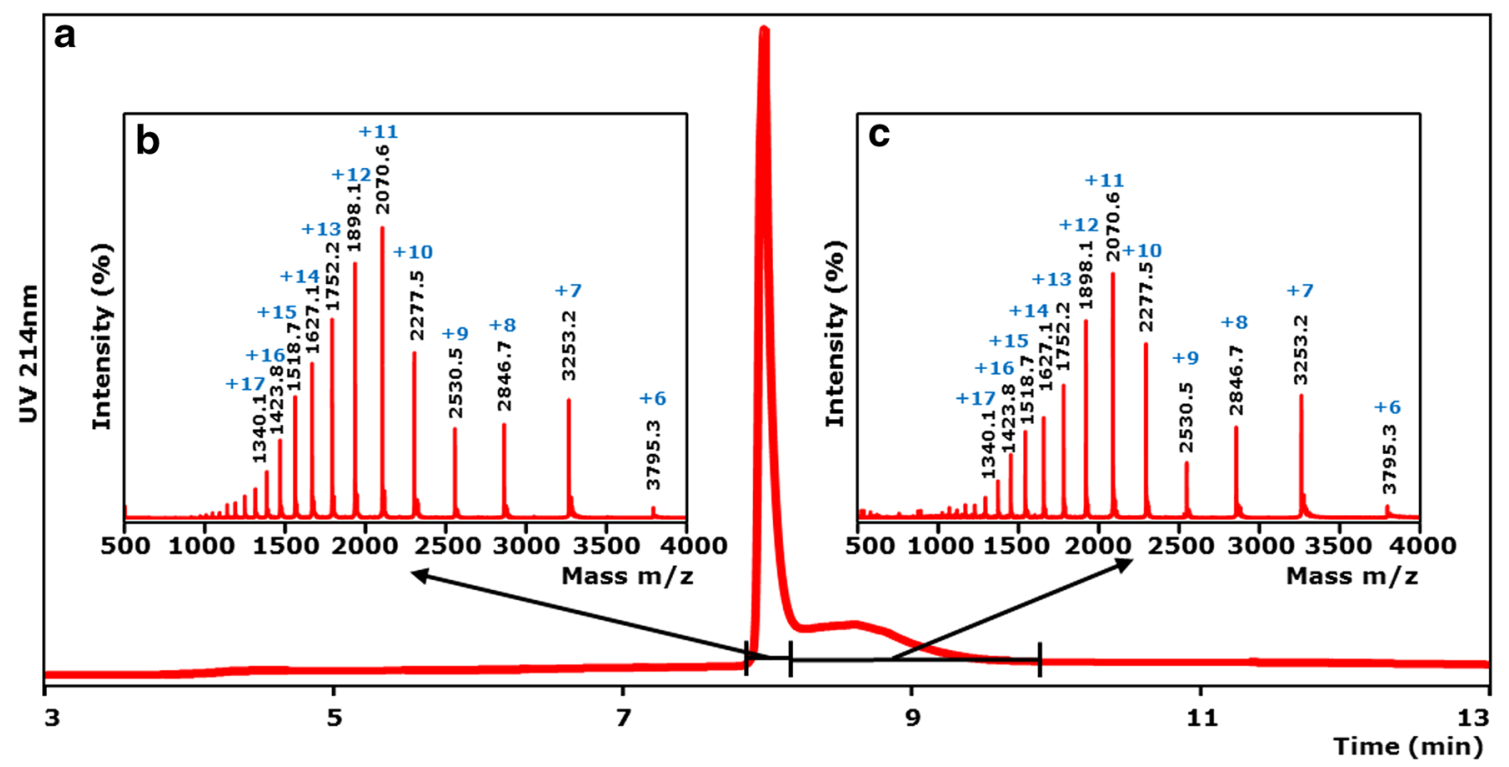

Figure 1 LC/ESI-MS analysis of MtLPMO9A. Purified MtLPMO9A was analyzed by LC/UV/ESI-MS using an ACQUITY UPLC separation system and a SYNAPT ion mobility mass spectrometer. a Chromatographic profile of purified MtLPMO9A (UV $214 \mathrm{~nm}$ ). ESI MS spectra (m/z values) of the main peak (b) and a shallow peak (c) observed by UV. The main and shallow peak showed exactly the same mass spectrum corresponding to the same protein with an $\mathrm{m} / \mathrm{z}$ of $22,765 \mathrm{Da}$. The main and shallow peak together featured about $99.5 \%$ of the total area measured in the UV trace at $214 \mathrm{~nm}$ and $94.7 \%$ of the total area measured in the total ion current (TIC) mass chromatogram (See "Methods"). Blue numbers represent different charge states of MtLPMO9A.

model (Figure 2) shows a highly conserved $\beta$-sheet core, whereas the loops differ from the TtPMO1 structure reported. The divalent metal ion in the active site is coordinated by the three amino acids, His1, His68 and Tyr153. The tyrosine (Tyr67) above the coordination site is typical for the PMO1 subgroup of the AA9 family [30]. Of the amino acids proposed to form the flat area of the TtPMO1 substrate-binding site, only one tyrosine is replaced by an asparagine in MtLPMO9A (Asn191). This tyrosine is also not conserved in other LPMO structures available in the Protein Data Bank. Based on the structural model shown in Figure 2, MtLPMO9A comprises two disulfide bridges, Cys126-Cys208 ( $-2 \mathrm{Da})$ and Cys38-Cys156 ( $-2 \mathrm{Da})$. The predicted mass of $M t$ LPMO9A (22,755.3 Da; amino acid sequence without signal peptide) is $10 \mathrm{Da}$ lower than the actual mass determined by ESI-MS (22,765.3 Da). Based on this calculation, $M t$ LPMO9A is expected to contain a methylated $\mathrm{N}$-terminal histidine (predicted mass from the amino acid sequence +14 Da for the methyl group bound to the histidine).

\section{Activity of MtLPMO9A on amorphous cellulose}

The activity of $M t$ LPMO9A was assayed on regenerated amorphous cellulose (RAC), both in the absence and presence of the external electron donor ascorbic acid. From HPAEC and MALDI-TOF MS analysis
(Figure 3), it can be concluded that in the presence of ascorbic acid, RAC is degraded by MtLPMO9A and that both $\mathrm{C} 1$ and $\mathrm{C} 4$ oxidized gluco-oligosaccharides $\left(\mathrm{GlcOS}_{\mathrm{n}}^{\#}\right.$ and $\mathrm{GlcOS}_{\mathrm{n}}{ }^{*}$, respectively) and non-oxidized gluco-oligosaccharides $\left(\mathrm{GlCOS}_{\mathrm{n}}\right)$ were formed. Furthermore, in the absence of ascorbic acid, no oxidized and non-oxidized gluco-oligosaccharides were found, which indicates that both hydrolytic and oxidative cleavage activity toward RAC are absent (Additional file 2). The annotation by HPAEC of oxidized gluco-oligosaccharides was performed using the published elution pattern of C1- and C4-oxidized gluco-oligosaccharides formed by NCU01050 or NCU08760 from Neurospora crassa [22, 31]. In addition, the formation of oxidized glucooligosaccharides was confirmed by the masses identified with MALDI-TOF MS (Figure 3c), based on previously proposed LPMO cleaving mechanisms [31-33]. In short, oxidation at $\mathrm{C} 1$ of the pyranose ring leads to formation of an unstable $\delta$-lactone, which in the presence of water converts to an aldonic acid. Lactone formation results in a 2 Da lower mass compared to the non-oxidized glucooligosaccharides, while aldonic acid formation results in a 16 Da higher mass (Figure 3b, c, marked with ${ }^{\#}$ ). Some of these acid groups may exchange an $\mathrm{H}$ ion for an $\mathrm{Li}$ ion, leading to a double $\mathrm{Li}$ adduct yielding an additional mass of $6 \mathrm{Da}$ (Figure 3c, marked with ${ }^{\S}$ ). Such double adducts have also been described for galacturonic acid 


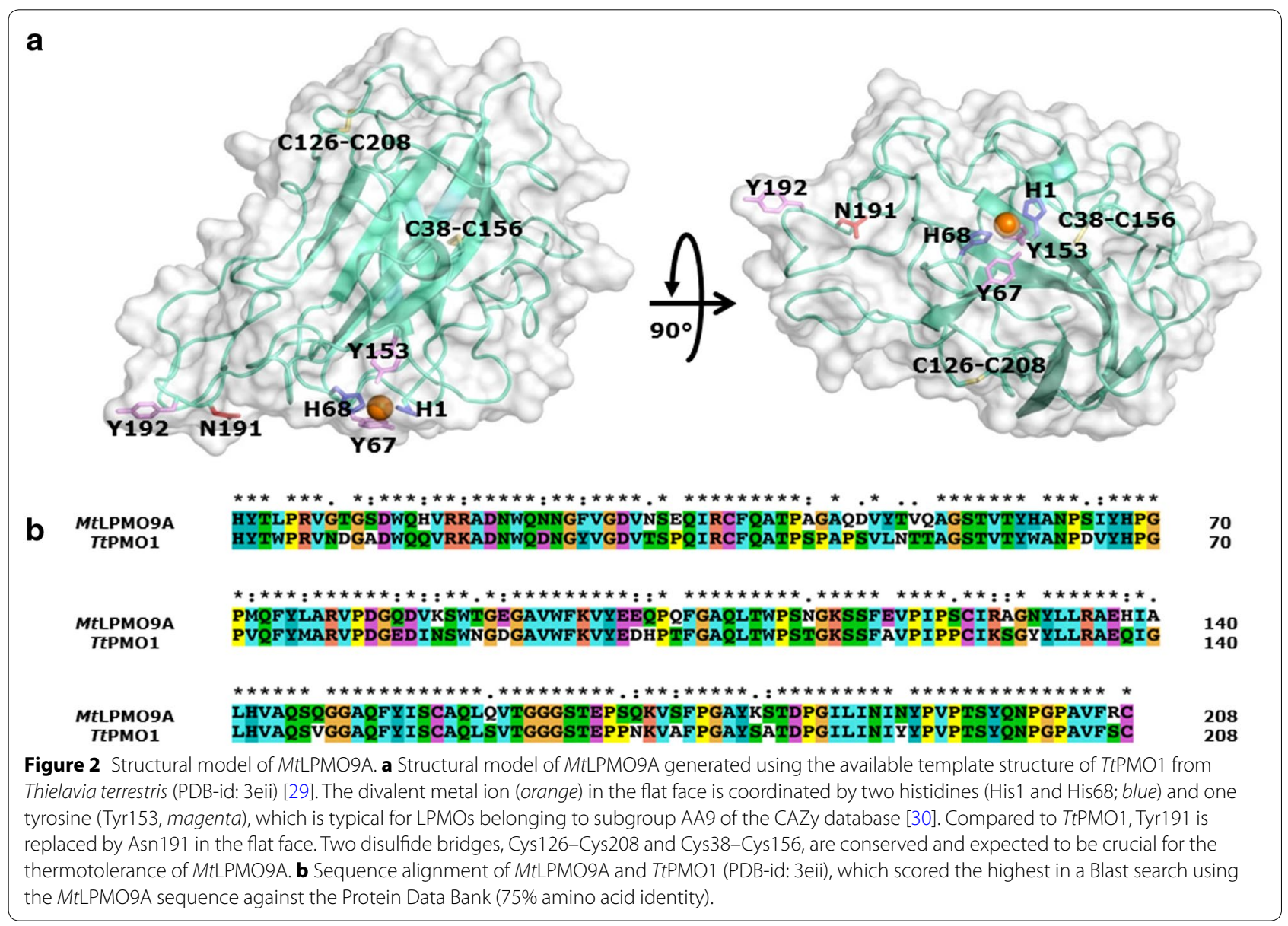

oligosaccharides in MALDI-TOF MS [34]. Similarly, oxidation at the $\mathrm{C} 4$ position leads to a 4-ketoaldose (Figure 3c, marked with *), which is rather stable. It corresponds, like the lactone, to a $2 \mathrm{Da}$ lower mass compared to the non-oxidized gluco-oligosaccharides [23, 32].

Unexpectedly, besides oxidized and non-oxidized gluco-oligosaccharides, also masses of $\mathrm{C} 1$ and $\mathrm{C} 4$ oxidized xylo-oligosaccharides $\left(\mathrm{XOS}_{\mathrm{n}}^{\#}\right.$ and $\mathrm{XOS}_{\mathrm{n}}{ }^{*}$, respectively) and non-oxidized xylo-oligosaccharides $\left(\mathrm{XOS}_{\mathrm{n}}\right)$ were observed (Figure 3c), although Avicel is known to contain around $2 \%(\mathrm{w} / \mathrm{w})$ of xylan [35]. This striking observation suggested that $M t$ LPMO9A is capable of oxidatively cleaving xylan next to cellulose. Based on the proposed LPMO cleaving mechanism for gluco-oligosaccharides as described above, the oxidation of the $\mathrm{C} 1$ and $\mathrm{C} 4$ position of xylo-oligosaccharides leads to a $2 \mathrm{Da}$ lower mass compared to non-oxidized xylo-oligosaccharides. Xylo-oligosaccharides oxidized at the $\mathrm{C} 1$ position are, like C1-oxidized gluco-oligosaccharides, unstable $\delta$-lactones, which hydrolyse in water to the corresponding aldonic acids $\left(\mathrm{XOS}_{\mathrm{n}}^{\#} ;+16 \mathrm{Da}\right)$.

\section{Activity of MtLPMO9A on three types of xylans}

The observation that MtLPMO9A generates oxidized xylo-oligosaccharides from RAC next to oxidized glucooligosaccharides is new and such an action of LPMOs has not been described for other LPMOs. Therefore, wheat arabinoxylan (WAX), birchwood glucuronoxylan (BiWX) and oat spelt xylan (OSX) were incubated with $M t$ LPMO9A in the absence or presence of ascorbic acid. The products formed were determined by using HPAEC and MALDI-TOF MS (Additional files 3, 4, 5). Remarkably, both in the absence and presence of ascorbic acid, no oxidized xylo-oligosaccharides were observed. In fact, non-oxidized xylo-oligosaccharides were released, which most likely pointed at the presence of a minor xylanolytic hydrolase impurity.

\section{Activity of MtLPMO9A on xylan-RAC mixtures}

Since $M t$ LPMO9A generated oxidized xylo-oligosaccharides from RAC next to oxidized gluco-oligosaccharides, but not if xylan as substrate was used alone, the mode of action of $M t$ LPMO9A on xylan-rich cellulosic biomass was further investigated. Hereto, RAC was 


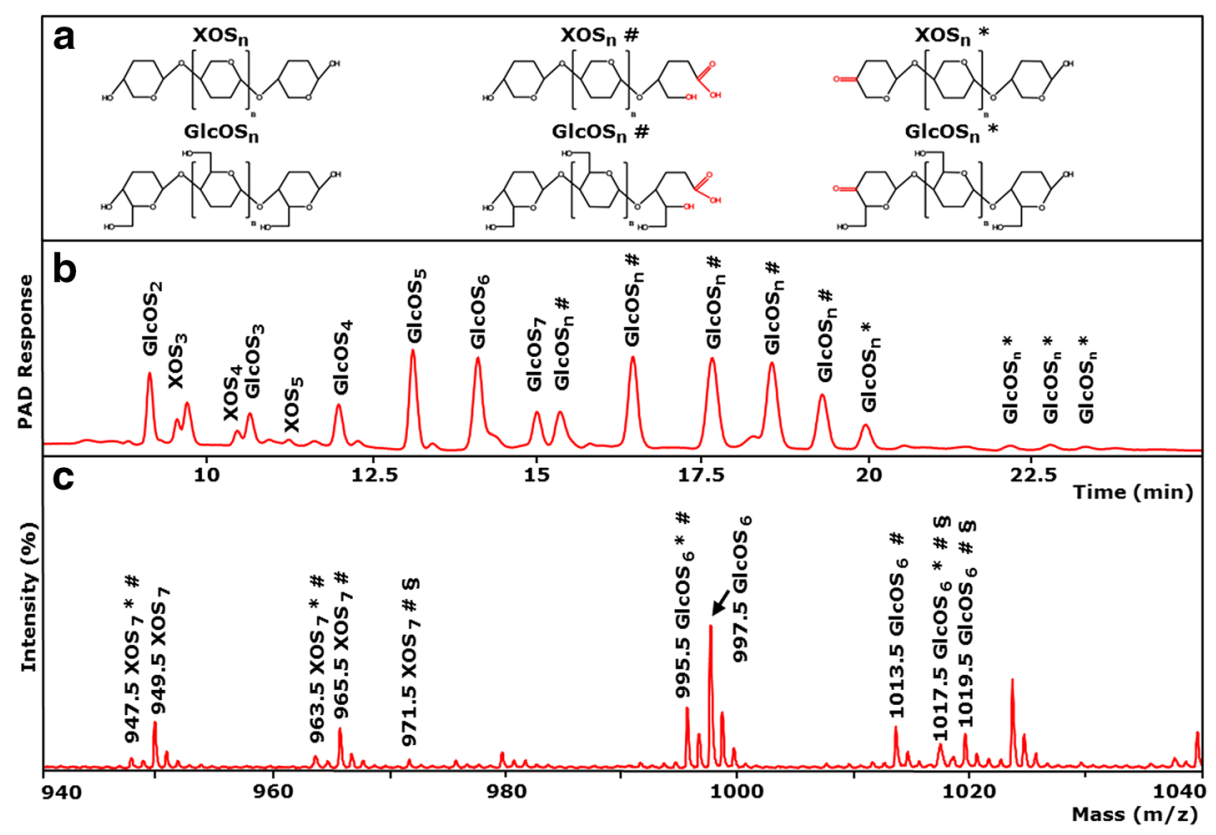

Figure 3 Activity of MtLPMO9A on amorphous cellulose. a Structure and nomenclature used: $X O S_{n}$ and GlcOS ${ }_{n}$, non-oxidized xylo- and glucooligosaccharides; $\mathrm{XOS}_{n}^{\#}$ and GlcOS $\#$, xylo- and gluco-oligosaccharides oxidized at the C1 carbon atom; $\mathrm{XOS}_{n}{ }^{*}$ and $\mathrm{GlcOS}_{n}{ }^{*}$, xylo- and gluco-oligosaccharides oxidized at the C4 carbon atom. b HPAEC elution pattern of regenerated amorphous cellulose (RAC) after incubation with MtLPMO9A ( $5 \mathrm{mg} \mathrm{g}^{-1}$ substrate). Samples were incubated in a $50 \mathrm{mM}$ ammonium acetate buffer ( $\mathrm{pH}$ 5.0) for $24 \mathrm{~h}$ at $52^{\circ} \mathrm{C}$ with ascorbic acid addition (1 mM). In the presence of ascorbic acid, oxidized GlCOS ${ }_{n}^{*}$ are formed by MtLPMO9A (marked either with * or *), of which the masses were analyzed by MALDI-TOF MS. Using RAC as a substrate, small amounts of non-oxidized XOS are detected by HPAEC. c MALDI-TOF mass spectrum of RAC incubated with MtLPMO9A with ascorbic acid. Clusters of oxidized $\mathrm{GlCOS}_{n}^{\# *}$ are determined as their lithium (Li) adducts. The insert shows masses of XOS ${ }_{n}^{\#}$ * and $\mathrm{GlCOS}_{n}^{\# *}$ oxidized either at C4 leading to a keto-group (* $-2 \mathrm{Da}$ ) or C1 leading to a lactone ( $\left.{ }^{*}-2 \mathrm{Da}\right)$. The $\delta$-lactones are unstable in water and hydrolyse to the corresponding aldonic acids ( ${ }^{\#}+16 \mathrm{Da}$ ). Double Li adducts (one Li adduct and one additional Li exchanged for $\mathrm{H}$ on the acid group) are C1-oxidized products $\left({ }^{\S}\right)$.

separately mixed with wheat arabinoxylan (WAX), birchwood glucuronoxylan (BiWX) or oat spelt xylan (OSX). $M t$ LPMO9A was added in the absence and presence of ascorbic acid. The products were determined using HPAEC and MALDI-TOF MS (Figures 4, 5; Additional file 5). In the absence of ascorbic acid, only non-oxidized xylo-oligosaccharides were formed from incubating RAC with BiWX, OSX or WAX. In the presence of ascorbic acid, however, the OSX-RAC and BiWX-RAC mixtures incubated with $M t$ LPMO9A showed formation of nonoxidized and oxidized xylo-oligosaccharides and of nonoxidized and oxidized GlcAme-xylo-oligosaccharides (4-O-methylglucuronic acid attached to xylo-oligosaccharides) next to non-oxidized and oxidized gluco-oligosaccharides (Figure 4). MALDI-TOF MS confirmed the formation of xylo-oligosaccharides and xylo-oligosaccharides oxidized at $\mathrm{C} 1\left(\mathrm{XOS}_{\mathrm{n}}^{\#}\right.$; $\left.+16 \mathrm{Da}\right)$ and at $\mathrm{C} 4\left(\mathrm{XOS}_{\mathrm{n}}{ }^{*}\right.$; $-2 \mathrm{Da})$. From the WAX-RAC mixture in the presence of ascorbic acid, the formation of oxidized and non-oxidized gluco-oligosaccharides was observed (Additional file 5), but not oxidized xylo-oligosaccharides.

\section{Synergy with EGI}

The synergy of $M t \mathrm{LPMO} 9 \mathrm{~A}$ with a pure EGI from Trichoderma viride $[36,37]$ in degrading RAC is shown in Figure 6. The release of $\mathrm{GlCOS}_{n}$ by EGI in the presence of $M t$ LPMO9A is around 16 and 8 times higher compared to the activity of pure EGI and pure $M t$ LPMO9A alone, respectively (based on the total HPAEC area of $\mathrm{GlcOS}_{2-5}$ ). The observed strong synergy between an LPMO and a cellulase has not been reported before.

\section{Discussion}

LPMOs constitute a new class of oxidative enzymes, which are expected to play a crucial role in the degradation of lignocellulosic biomass [23-25, 27]. We purified a new LPMO from the commercially applied fungus Myceliophthora thermophila $\mathrm{C} 1$ and investigated its degradation capacity on a wide range of substrates (Table 1). We show for the first time an LPMO that is able to oxidize substrates with a $\beta$-(1 $\rightarrow$ 4)-linked xylan backbone, in the presence of cellulose and the electron donor ascorbic acid. 


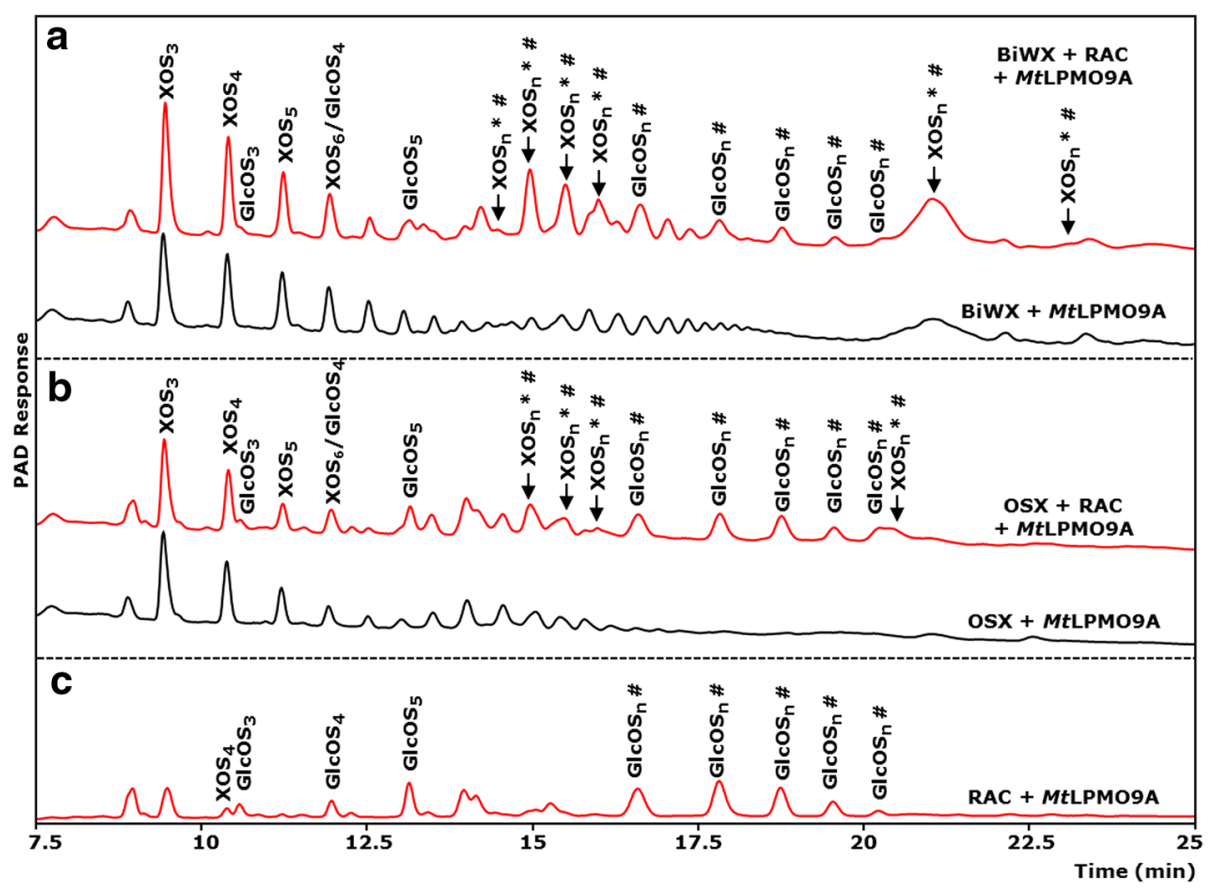

Figure 4 HPAEC elution pattern of xylan-RAC mixtures incubated with MtLPMO9A. a Birchwood xylan (BiWX) and b oat spelt xylan (OSX) ( $2 \mathrm{mg} \mathrm{mL}^{-1}$ ) in the presence and absence of regenerated amorphous cellulose (RAC; $2 \mathrm{mg} \mathrm{mL}^{-1}$ ) after incubation with MtLPMO9A (12.5 mg g-1 substrate). c HPAEC elution pattern of RAC after incubation with MtLPMO9A (12.5 $\mathrm{mg} \mathrm{g}^{-1}$ substrate). Samples were incubated in a $50 \mathrm{mM}$ ammonium acetate buffer ( $\mathrm{pH}$ 5.0) with ascorbic acid addition (1 mM). Incubation with MtLPMO9A of the two xylans and xylan-RAC mixtures, in the presence of ascorbic acid, results in the formation of non-oxidized linear xylo-oligosaccharides $\left(\mathrm{XOS}_{n}\right)$ and substituted xylo-oligosaccharides. Incubation of xylan-RAC mixtures with MtLPMO9A in the presence of ascorbic acid results in the formation of non-oxidized gluco-oligosaccharides (GlcOS ${ }_{n}$ ) and oxidized gluco-oligosaccharides (GlcOS $\#$ ). The incubation of MtLPMO9A with BiWX-RAC and OSX-RAC mixture in the presence of ascorbic acid results in the formation of numerous products (black arrow, indicated as oxidized xylo-oligosaccharides XOS ${ }_{n}^{*}$ ), which are not present if MtLPMO9A was incubated with BiWX, OSX or RAC alone. The results of MALDI-TOF MS analysis of BiWX-RAC and OSX-RAC mixture incubated with MtLPMO9A in the presence of ascorbic acid are shown in Figure 5.

For the formation of oxidized xylo-oligosaccharides by $M t$ LPMO9A in the presence of ascorbic acid, the addition of cellulose to xylans is essential. During incubations without ascorbic acid no oxidized xylo- and gluco-oligmers were detected. We considered the idea that the formation of glucyl radical intermediates of cellulose by $M t$ LPMO9A [31, 32] might have oxidized the xylan backbone. This hypothesis, however, seemed to be unlikely since also other LPMOs cleave cellulose under the formation of oxidized gluco-oligosaccharides via glucyl radical intermediates, but the formation of oxidized xylo-oligosaccharides has not been reported [23, 28]. We showed that the oxidized xylo-oligosaccharides detected were not from RAC only. Specifically, oxidized GlcAme-xylo-oligosaccharides from the cleavage of BiWX and OSX were also detected, but only if RAC was incubated together with BiWX or OSX (Figures 4, 5). Hence, we hypothesize that MtLPMO9A uses the cellulose to bind while oxidizing neighboring xylan chains. This idea is strengthened by the observation that in contrast to RAC-BiWX and RAC-OSX mixtures, oxidized xylo-oligosaccharides are not formed with RAC-WAX mixtures. Unlike WAX, both BiWX and OSX consist of large sequences of unsubstituted xylosyl residues [4, 38]. Such long linear unsubstituted xylans are reported to be associated with cellulose via hydrogen bonding [2, 4]. WAX, on the other hand, has a high degree of arabinosyl substituents present on the $\beta$-(1 $\rightarrow 4)$-linked xylan backbone [39], which has been shown to prevent association with cellulose chains [4]. Here, we show for the first time an LPMO, which benefits from the strong interaction between low substituted linear xylan and cellulose. The discovered activity of $M t$ LPMO9A provides a new paradigm in the understanding of the degradation of xylan-coated cellulose.

Recently, NcLPMO9C from $N$. crassa expressed in P. pastoris was described to have an activity on hemicellulosic $\beta-(1 \rightarrow 4)$-linked glucans [24]. We found that MtLPMO9A showed a similar mode of action on hemicellulosic $\beta$ - $(1 \rightarrow 4)$-linked glucans and $\beta-(1 \rightarrow 3$, $1 \rightarrow 4$ )-linked glucans (Additional files 6, 7). However, formation of oxidized xylo-oligosaccharides so far has only been observed for $M t$ LPMO9A. 


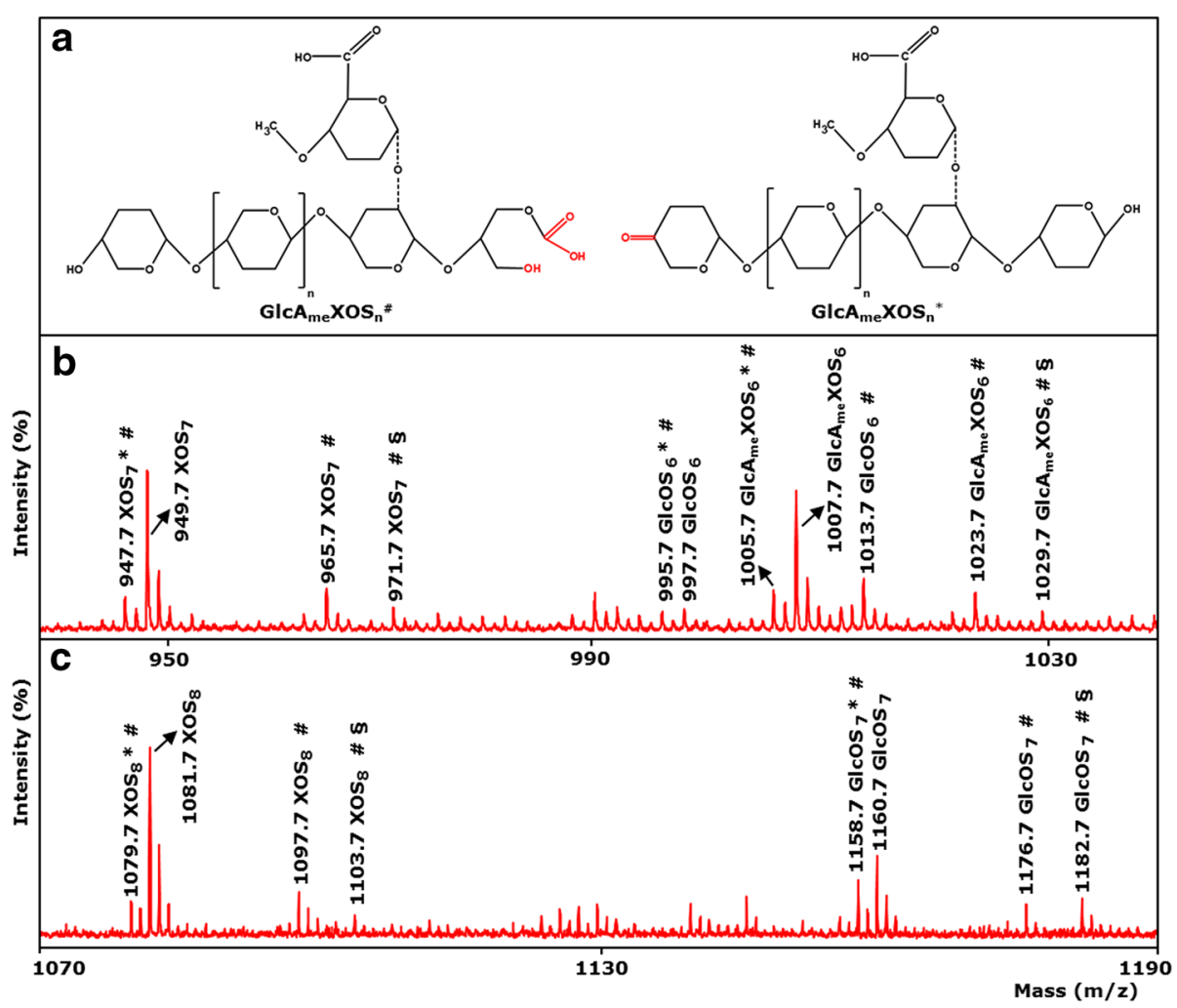

Figure 5 MALDI-TOF MS spectra of xylan-RAC mixtures incubated with MtLPMO9A. b Birchwood xylan (BiWX; $2 \mathrm{mg} \mathrm{mL}^{-1}$ ) and $\mathbf{c}$ oat spelt xylan (OSX; $2 \mathrm{mg} \mathrm{mL}^{-1}$ ) in the presence of regenerated amorphous cellulose (RAC; $2 \mathrm{mg} \mathrm{mL}^{-1}$ ) after incubation of MtLPMO9A (10 mg g $\mathrm{m}^{-1}$ substrate). Samples were incubated in a $50 \mathrm{mM}$ ammonium acetate buffer ( $\mathrm{pH} \mathrm{5.0)}$ for $24 \mathrm{~h}$ at $52^{\circ} \mathrm{C}$ with ascorbic acid addition (1 mM). MtLPMO9A incuba-

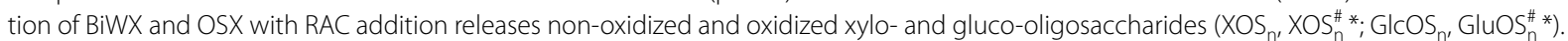
The presence of C4-oxidized $\mathrm{XOS}_{n}{ }^{*}$, and $\mathrm{XOS}_{n}^{\#}$ oxidized at $\mathrm{C} 1$ to an aldonic acid (\# $+16 \mathrm{Da}$ ) is shown. Non-oxidized GlcOS $\mathrm{S}_{n}$ and oxidized GlcOS ${ }_{n}^{*}$ * are less detectable due to abundance of xylo-oligosaccharides present. From BiWX also 4-O-methylglucoronic acid containing non-oxidized XOS ${ }_{n}$

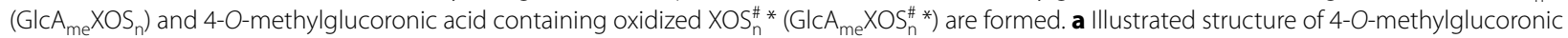
acid containing $\mathrm{C1}$ - and C4-oxidized $\mathrm{XOS}_{n}$. $\left(\mathrm{GlCA}_{m e} \mathrm{XOS}_{n^{\prime}}^{\#} \mathrm{GlCA}_{m e} \mathrm{XOS}_{n}{ }^{*}\right.$, respectively). Masses represent lithium adducts only. Double Li adducts are determined for C1-oxidized products ( ${ }^{\S}+6 \mathrm{Da}$ ). MALDI-TOF MS analysis of BiWX and OSX in the presence of RAC after incubation of MtLPMO9A without ascorbic acid did not reveal detectable amounts of oxidized products (data not shown).

Based on their amino acids in the substrate-binding site, LPMOs of the AA9 class are further divided into the subgroups, PMOI, PMOII and PMOIII [30]. MtLPMO9A shows most similarity with subgroup PMOI and has the highest amino acid sequence identity with TtPMO1 (75\%). Like TtPMO1 [29], MtLPMO9A considerably enhances glucose release from cellulose when added to a cellulase cocktail. Additionally, $M t$ LPMO9A shows a strong synergistic effect with EGI on amorphous cellulose as shown in the present study. During enzyme purification, the oxidative activity of $M t$ LPMO9A was separated from a strong hydrolytic activity toward cellulose (data not shown). Probably, $M t$ LPMO9A and the enzyme responsible for this hydrolytic activity closely work together in vivo (Additional file 8). Possibly, during the evolution of fungi, the development of enzymes containing both oxidative activities and synergism with hydrolases enabled a more efficient degradation of a wider range of substrates present in nature.

\section{Conclusion}

The enzymatic degradation of cellulose and xylan-rich agricultural and industrial food by-products into monosaccharides is seen as a basis for the production of biofuels and bio-based chemicals. Now, for the first time, we demonstrate the activity of a lytic polysaccharide monooxygenase (MtLPMO9A) that shows oxidative cleavage of xylan in addition to cellulose and that acts in synergism with endoglucanase I. The ability of $M t \mathrm{LP}-$ MO9A to cleave the xylan-coated cellulose regions is considered to be important for loosening the rigid plant polysaccharide matrix in plant biomass, enabling an increased accessibility for hydrolytic enzymes. This discovery provides new insights into how fungi degrade 


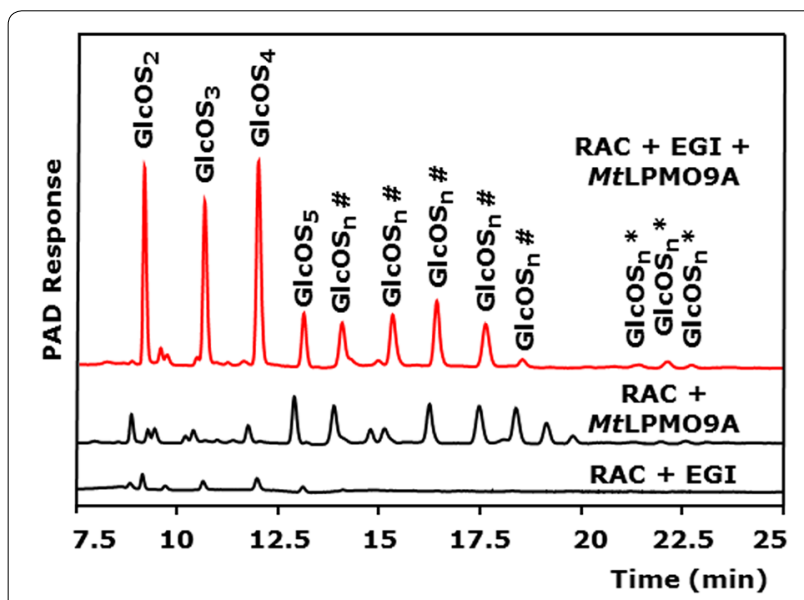

Figure 6 HPAEC elution patterns of RAC incubated with MtLPMO9A and EGI. Regenerated amorphous cellulose (RAC, $2 \mathrm{mg} \mathrm{mL}^{-1}$ ) before and after incubation with MtLPMO9A (10 $\mathrm{mg} \mathrm{g}^{-1}$ substrate) and/or endoglucanase I from T. viride (EGI) (100 $\mathrm{g} \mathrm{g} \mathrm{g}^{-1}$ substrate). Samples were incubated in a $50 \mathrm{mM}$ ammonium acetate buffer ( $\mathrm{pH}$ 5.0) for $24 \mathrm{~h}$ at $52^{\circ} \mathrm{C}$ with ascorbic acid addition $(1 \mathrm{mM})$. In the presence of ascorbic acid, mainly oxidized gluco-oligosaccharides (GlcOS ${ }_{n}^{*}$ ) are formed by MtLPMO9A from RAC (marked either with " for C1 or * for (4 oxidation). Incubation of EGI with RAC results in hardly detectable non-oxidized gluco-oligosaccharides (DP2-5). The combined addition of $\mathrm{EGI}$ and MtLPMO9A results in a 16-fold higher release of nonoxidized $\mathrm{GlCOS}_{n}$ (based on comparison of the sum of AUC of $\mathrm{GlCOS}_{2-4}$ determined by HPAEC) from RAC compared to EGl incubated with RAC only.

plant cell wall structures by using both oxidative activity and synergism with hydrolases and, in addition, how to boost hydrolytic enzyme cocktails for biorefinery applications.

\section{Methods}

Enzyme expression, production and purification

MtLPMO9A from Myceliophthora thermomphila C1 (UniProt: KP901251) was over-expressed in a protease/ (hemi-) cellulase-free C1-expression host (LC strain) [40, 41]. The $\mathrm{C} 1$ strain was grown aerobically in 2-L fermentors using a medium containing glucose and ammonium sulfate, and enriched with essential salts [41]. Enzyme production was performed under glucose limitation in a fed-batch process $\left(\mathrm{pH} 6.0 ; 32^{\circ} \mathrm{C}\right)$ as described previously [40] and resulted in an $M t$ LPMO9A-rich crude enzyme extract. The crude enzyme extract was dialyzed against $10 \mathrm{mM}$ potassium phosphate buffer ( $\mathrm{pH}$ 7.0). MtLPMO9A was purified using an AKTA-Explorer preparative chromatography system (GE Healthcare, Uppsala, Sweden). As a first step, $3 \mathrm{~g}$ of the dialyzed crude enzyme mixture $\left(50 \mathrm{mg} \mathrm{mL}^{-1}\right.$ ) was subjected to a self-packed Source 15Q column $(100 \times 70 \mathrm{~mm}$ internal diameter, GE Healthcare), pre-equilibrated in $20 \mathrm{mM}$ potassium phosphate buffer ( $\mathrm{pH}$ 7.0). After protein application,
Table 1 MtLPMO9A oxidation on various polysaccharide substrates

\begin{tabular}{|c|c|c|c|c|}
\hline \multirow[t]{3}{*}{ Substrate } & \multicolumn{4}{|c|}{ Occurrence of oxidation } \\
\hline & \multicolumn{2}{|c|}{ Without ascorbic acid } & \multicolumn{2}{|c|}{$\begin{array}{l}\text { With } 1 \mathrm{mM} \text { ascor- } \\
\text { bic acid }\end{array}$} \\
\hline & $\mathrm{GlcOS}_{n}^{\# * a}$ & $\operatorname{xOS}_{n}^{\# * b}$ & $\operatorname{GlcOS}_{n}^{\#}$ & $\operatorname{xOS}_{n}^{\# *}$ \\
\hline \multicolumn{5}{|l|}{ Cellulose } \\
\hline Avicel $^{c}$ & - & - & + & + \\
\hline $\operatorname{RAC}^{\mathrm{C}}$ & - & - & + & + \\
\hline \multicolumn{5}{|l|}{ Hemicellulose } \\
\hline \multicolumn{5}{|l|}{ Glucan } \\
\hline Xyloglucan $^{d}$ & - & - & + & - \\
\hline$\beta$-Glucan barley & - & - & + & - \\
\hline$\beta$-Glucan oat spelt & - & - & + & - \\
\hline \multicolumn{5}{|l|}{ Xylan } \\
\hline osx & - & - & - & - \\
\hline BiWXe & - & - & - & - \\
\hline WAX & - & - & - & - \\
\hline \multicolumn{5}{|l|}{ Oligosaccharides } \\
\hline Gluco-oligosaccharides ${ }^{f}$ & - & - & - & - \\
\hline Xylo-oligosaccharides ${ }^{f}$ & - & - & - & - \\
\hline Galactomannan ${ }^{g}$ & - & - & - & - \\
\hline \multicolumn{5}{|c|}{ RAC/hemicellulose combination } \\
\hline $\mathrm{RAC}+\mathrm{BiWX}$ & - & - & + & + \\
\hline RAC + OSX & - & - & + & + \\
\hline RAC + WAX & - & - & - & - \\
\hline \multicolumn{5}{|c|}{ a Gluco-oligosaccharides oxidized at the $\mathrm{C} 1\left(\mathrm{GlcOS}_{n}^{\#}\right)$ or $\mathrm{C} 4$ position $\left(\mathrm{GlcOS}_{n}{ }^{*}\right)$} \\
\hline \multicolumn{5}{|c|}{ b Xylo-oligosaccharides oxidized at the $\mathrm{C} 1\left(\mathrm{XOS}_{n}^{\#}\right)$ or C4 position $\left(\mathrm{XOS}_{n}{ }^{*}\right)$} \\
\hline \multicolumn{5}{|c|}{ c Regenerated amorphous cellulose (RAC), crystalline cellulose (Avicel). } \\
\hline \multicolumn{5}{|c|}{ d Xyloglucan from tamarind seed. } \\
\hline \multicolumn{5}{|c|}{ e Oat spelt xylan (OSX), birchwood xylan (BiWX), wheat arabinoxylan (WAX). } \\
\hline \multicolumn{5}{|c|}{$\begin{array}{l}f \beta-(1 \rightarrow 4) \text {-linked gluco-and xylo-oligosaccharides, degree of polymerization } \\
2-5 .\end{array}$} \\
\hline $\begin{array}{l}{ }^{g} \beta-(1 \rightarrow 4)-\text {-linked-D-mannos } \\
\text { purchased from Megazyme }(\mathrm{E}\end{array}$ & syl backbone & om guar (mec & dium viscosit & \\
\hline
\end{tabular}

the column was washed with three column volumes of $20 \mathrm{mM}$ potassium phosphate buffer $(\mathrm{pH}$ 7.0). Elution was performed with a linear gradient of $0-1 \mathrm{M} \mathrm{NaCl}$ in $20 \mathrm{mM}$ potassium phosphate buffer ( $\mathrm{pH}$ 7.0) over five column volumes at $25 \mathrm{~mL} \mathrm{~min}^{-1}$. The eluate was monitored at 220 and $280 \mathrm{~nm}$. Fractions $(20 \mathrm{~mL})$ were collected and immediately stored on ice. Peak fractions were pooled and concentrated using ultrafiltration (Amicon Ultra, molecular mass cut-off of $3 \mathrm{kDa}$, Merck Millipore, Cork, Ireland) at $4^{\circ} \mathrm{C}$. The concentrated pools were subjected to SDS-PAGE (Additional file 1). For further purification (2nd step), the MtLPMO9A-containing pool (fraction AEC-I, Additional file 1) was loaded onto a selfpacked Superdex TM-75 column $(100 \times 3 \mathrm{~cm}$ internal diameter, GE Healthcare) and eluted at $5 \mathrm{~mL} \mathrm{~min}^{-1}$ with a $10 \mathrm{mM}$ potassium phosphate buffer $(\mathrm{pH} 7.0)$ containing 
$150 \mathrm{mM} \mathrm{NaCl}$. Fractions $(5 \mathrm{~mL})$ were immediately stored on ice. Peak fractions were pooled and concentrated by ultrafiltration as described above.

The $M t$ LPMO9A preparation thus obtained (partially purified fraction SEC-I; Additional file 1) was further subjected (3rd step) to a Resource Q column $(30 \times 16 \mathrm{~mm}$ internal diameter, GE Healthcare), pre-equilibrated in $20 \mathrm{mM}$ potassium phosphate buffer ( $\mathrm{pH}$ 7.0). After protein application, the column was washed with 20 column volumes of starting buffer. Elution at $6 \mathrm{~mL} \mathrm{~min}{ }^{-1}$ was performed with a linear gradient of $0-1 \mathrm{M} \mathrm{NaCl}$ in $20 \mathrm{mM}$ potassium phosphate buffer ( $\mathrm{pH} 7.0$ ) over 20 column volumes. Elution was monitored at 220 and $280 \mathrm{~nm}$. Fractions $(3 \mathrm{~mL})$ were immediately stored on ice. Peak fractions were pooled and concentrated by ultrafiltration as described above.

\section{Protein identification}

Sequencing of the $M t \mathrm{LPMO} 9 \mathrm{~A}$ coding sequence was carried out by the Scripps Research Institute, USA.

\section{Protein content}

To analyze protein contents, the BCA Protein Assay Kit (Thermo Scientific, Rockford, IL, USA) was used with bovine serum albumin (BSA) as calibration.

\section{SDS-PAGE}

The protein purity was analyzed by using sodium dodecyl sulfate-polyacrylamide gel electrophoresis (SDS-PAGE). Therefore, proteins were reduced with $\beta$-mercaptoethanol, heated for $10 \mathrm{~min}$ and loaded on $12 \%$ polyacrylamide gels (Mini-PROTEAN TGX Gels, BioRad Laboratories, Hempel Hempstead, UK). In addition, a protein marker (Protein All Blue Standards, Bio-Rad Laboratories) was loaded for mass calibration. Gels were stained with the EZBlue Gel Staining Reagent (Sigma Aldrich, Steinheim, Germany).

\section{LC/ESI-MS}

Purified MtLPMO9A (2.5 mg mL $\mathrm{m}^{-1}$ in $0.1 \%(\mathrm{v} / \mathrm{v})$ trifluoroacetic acid (TFA) in $\mathrm{H}_{2} \mathrm{O}$ ) was analyzed by liquid chromatography/electron spray ionization-mass spectrometry (LC/ESI-MS) using an ACQUITY UPLC separation system (Waters, Milford, MA, USA) equipped with a C4-reversed phase column (UPLC BEH C4 $1.7 \mu \mathrm{m}$, $2.1 \times 100 \mathrm{~mm}$, Waters) coupled to a PLC LG 500 photodiode array detector (Waters) and a SYNAPT G2-Si High Definition Mass Spectrometer (Waters). Gradient elution with eluent $\mathrm{A}\left(\mathrm{H}_{2} \mathrm{O}+1 \%(\mathrm{v} / \mathrm{v})\right.$ acetonitrile $+0.1 \%$ $(\mathrm{v} / \mathrm{v})$ TFA) and eluent B (acetonitrile $+0.1 \%(\mathrm{v} / \mathrm{v})$ TFA) was performed according to the following steps: From 0 to $2 \mathrm{~min}$ isocratic $90 \% \mathrm{~A}$, from 2 to $12 \mathrm{~min}$ gradient from $90 \%$ A to $25 \%$ A, from 12 to 15 min gradient from $25 \%$ A to $100 \% \mathrm{~B}$ and from 12 to $15 \mathrm{~min}$ isocratic at $100 \% \mathrm{~B}$; then re-equilibration to the initial conditions. The flow rate and the injection volume were $0.35 \mathrm{~mL} \mathrm{~min}^{-1}$ and $2 \mu \mathrm{L}$, respectively. The photodiode array detector was operated at a sampling rate of 40 points $\mathrm{sec}^{-1}$ in the range 200$400 \mathrm{~nm}$, resolution $1.2 \mathrm{~nm}$. The SYNAPT mass spectrometer was operated in the positive ion mode (resolution mode), capillary voltage $3 \mathrm{kV}$, sampling cone $30 \mathrm{~V}$, source temperature $150^{\circ} \mathrm{C}$, desolvation temperature $500^{\circ} \mathrm{C}$, cone gas flow $\left(\mathrm{N}_{2}\right) 200 \mathrm{~L} \mathrm{~h}^{-1}$, desolvation gas flow $\left(\mathrm{N}_{2}\right) 800$ $\mathrm{L} \mathrm{h}^{-1}$, acquisition in the full scan mode, scan time $0.3 \mathrm{~s}$, interscan time $0.015 \mathrm{~s}$, acquisition range $150-4,000 \mathrm{~m} / z$.

\section{Substrates incubated with MtLPMO9A}

OSX, BiWX, Avicel PH-101, xylo-oligosaccharides (DP1$5)$ and $\beta-(1 \rightarrow 4)$-linked gluco-oligosaccharides (DP1-5) were obtained from Sigma-Aldrich (Steinheim, Germany). WAX (medium viscosity), $\beta$-( $1 \rightarrow 3,1 \rightarrow 4$ )-linked glucan from barley (medium viscosity) and oat spelt (medium viscosity) were purchased from Megazyme (Bray, Ireland). Xyloglucan (XG; from tamarind seed) was obtained from Dainippon Sumitomo Pharma (Osaka, Japan). Regenerated amorphous cellulose (RAC) was prepared from Avicel $\mathrm{PH}-101$ by adopting a method described elsewhere [42]. Briefly, Avicel PH-101 (100 mg) was moistened with $0.6 \mathrm{~mL}$ water. Next, $10 \mathrm{~mL} \mathrm{86.2 \%} \mathrm{(w/v)} \mathrm{ortho-phosphoric}$ acid was slowly added followed by rigorous stirring for 30 min until the cellulose was completely dissolved. The dissolved cellulose precipitated during stepwise addition of $40 \mathrm{~mL}$ of water. After centrifugation $(4,000 \mathrm{~g}, 12 \mathrm{~min}$, $4^{\circ} \mathrm{C}$ ), the pellet obtained was washed twice with water and neutralized ( $\mathrm{pH} 7.0$ ) with $2 \mathrm{M}$ sodium carbonate. The pellet was washed again with water (three times) and the final pellet was suspended in water to a dry matter content of $1.4 \pm 0.1 \%(\mathrm{w} / \mathrm{w})$ RAC suspension.

\section{MtLPMO9A activity assays}

Substrates (1-2 mg mL $\mathrm{mL}^{-1}$, see Figure captions) were dissolved in $50 \mathrm{mM}$ ammonium acetate buffer ( $\mathrm{pH}$ 5.0), with or without addition of ascorbic acid (final concentration of $1 \mathrm{mM}) . M t$ LPMO9A was added $\left(12.5 \mu \mathrm{g} \mathrm{mg}{ }^{-1}\right.$ substrate) and incubated for $24 \mathrm{~h}$ at $50^{\circ} \mathrm{C}$ in a head-over-tail rotator in portions of $1 \mathrm{~mL}$ total volume (Stuart rotator, Bibby Scientific, Stone, UK) at $20 \mathrm{rpm}$. Supernatants of all incubations, including substrates incubated with and without ascorbic acid in the absence of MtLPMO9A, were analyzed by HPAEC and MALDI-TOF MS.

\section{Structural modelling}

An alignment was made of the amino acid sequence of $M t$ LPMO9A and the amino acid sequence of PMO1 from Thielavia terrestris, which scored highest in a Blast search using the $M t$ LPMO9A sequence against the 
Protein Data Bank (75\% amino acid identity). Using this alignment and the available structure of TtPMO1 (PDBid: 3eii) as template, structural models were obtained for $M t$ LPMO9A using the Modeller program version 9.14 [43]. Thirty comparative models were generated, after which the model with the lowest corresponding DOPE score [44] was selected for image generation using Pymol (Pymol, The PyMOL Molecular Graphics System, Version 1.5.0.4 Schrödinger, LLC, New York, NY, USA).

\section{Oligosaccharides analysis}

Oligosaccharides were analyzed by high-performance anion exchange chromatography (HPAEC) with pulsed amperometric detection (PAD). The HPAEC system (ICS-5000, Dionex, Sunnyvale, CA, USA) was equipped with a combination of a CarboPac PA1 guard column (50 $\mathrm{mm} \times 2 \mathrm{~mm}$ i.d., Dionex) and a CarboPac PA1 analytical column $(250 \mathrm{~mm} \times 2 \mathrm{~mm}$ i.d., Dionex). The flow rate was $0.3 \mathrm{~mL} \mathrm{~min}^{-1}\left(20^{\circ} \mathrm{C}\right)$. Samples were kept at $6^{\circ} \mathrm{C}$ in the autosampler and the injection volume was $10 \mu \mathrm{L}$. Elution was performed using two mobile phases: $0.1 \mathrm{M}$ $\mathrm{NaOH}$ and $1 \mathrm{M} \mathrm{NaOAc}$ in $0.1 \mathrm{M} \mathrm{NaOH}$. The gradient elution program was as follows: $0-30 \mathrm{~min}$, linear gradient 0-400 mM NaOAc; 30-40 min linear gradient $400-1,000 \mathrm{mM} \mathrm{NaOAc}$; followed by a cleaning step and equilibration (15 $\mathrm{min}$ ) of the column with the starting conditions. Soluble gluco- and xylo-oligosaccharides (degree of polymerization 1-5) as well as glucuronic and gluconic acid were used as standards (Sigma-Aldrich).

\section{MALDI-TOF MS}

For matrix-assisted laser desorption ionization-time of flight mass spectrometry (MALDI-TOF MS), an Ultraflex workstation using FlexControl 3.3 (Bruker Daltonics) equipped with a nitrogen laser of $337 \mathrm{~nm}$ was used. The pulsed ion extraction was set on 80 ns. Ions were accelerated to a kinetic energy of $25 \mathrm{kV}$ and detected in positive reflector mode with a set reflector voltage of $26 \mathrm{kV}$. The lowest laser energy required was used to obtain a good signal-to-noise ratio. A total of 200 spectra were collected for each measurement. The mass spectrometer was calibrated using a mixture of maltodextrins (Avebe, Veendam, The Netherlands) in a mass range $(\mathrm{m} / \mathrm{z})$ of $500-2,500$. The peak spectra were processed by using FlexAnalysis software version 3.3 (Bruker Daltonics). Prior to analysis, samples were desalted by adding AG 50 W-X8 Resin (Bio-Rad Laboratories). To obtain lithium (Li) adducts, the supernatant was dried under nitrogen and re-suspended in $20 \mathrm{mM} \mathrm{LiCl}$ [28]. Each lithium-enriched sample of a volume of $1 \mu \mathrm{L}$ was mixed with $1 \mu \mathrm{L}$ of matrix solution $\left(12 \mathrm{mg} \mathrm{mL}^{-1} 2,5\right.$-dihydroxy-benzoic acid (Bruker Daltonics) in 30\% (v/v) acetonitrile in $\mathrm{H}_{2} \mathrm{O}$ ), applied on an MTP 384 massive target plate (Bruker Daltonics) and dried under a stream of warm air.

\section{Additional files}

Additional file 1: Figure 1. The purification of LPMO9A from Myceliophthora thermophila C1. A - Anion exchange chromatography (AEC) elution profile (step 1) of crude enzyme extract containing expressed MtLPMO9A. B - Size exclusion chromatography (SEC) elution profile of Pool AEC-I (step 2). The framed columns indicate the MtLPMO9A-containing fractions pooled and concentrated for further analysis. C - SDS-PAGE of marker (lane 1; Precision Plus Protein, Bio-Rad Laboratories), the crude enzyme extract (lane 2), pooled fraction AEC-I (lane 3) and partially purified fraction SEC-I (lane 4). Protein bands corresponding to MtLPMO9A are indicated by an arrow. For more details about protein purification see Materials and Methods.

Additional file 2: Figure 2. The HPAEC elution patterns and MALDI-TOF mass spectrum of cellulose incubated with MtLPMO9A. A - Regenerated amorphous cellulose (RAC; $2 \mathrm{mg} \mathrm{mL}^{-1}$ ) before and after incubation with MtLPMO9A (12.5 $\mathrm{mg} \mathrm{g}^{-1}$ substrate). Samples were incubated in a $50 \mathrm{mM}$ ammonium acetate buffer ( $\mathrm{pH}$ 5.0) for $24 \mathrm{~h}$ at $52^{\circ} \mathrm{C}$, either with ascorbic acid addition ( $1 \mathrm{mM}$ ) or without. In the presence of MtLPMO9A and ascorbic acid, non-oxidized gluco-oligomers ( $\left(\mathrm{ClOS}_{n}\right)$ and gluco-oligomers oxidized at C1 (GlcOS $\left.{ }_{n}^{\#}\right)$ and C4 (GlcOS $\left.{ }_{n}^{*}\right)$ are formed from RAC. Neither non-oxidized nor oxidized gluco-oligomers were formed by MtLPMO9A in the absence of ascorbic acid. In both incubations of RAC with MtLPMO9A, either with or without ascorbic acid, traces of non-oxidized xylo-oligomers were formed $\left(\mathrm{XOS}_{n}\right)$. B - MALDI-TOF mass spectrum of RAC incubated with MtLPMO9A with ascorbic acid. Clusters of oxidized gluco-oligomers are determined as their lithium (Li) adducts. See Figure 3 for more details.

Additional file 3: Figure 3. The MALDI-TOF MS analysis of xylan incubated with MtLPMO9A. (A) Wheat arabinoxylan (WAX), (B) birchwood (BiWX) and (C) oat spelt (OSX) xylan (2 $\mathrm{mg} \mathrm{mL}^{-1}$ ) after incubation with MtLPMO9A (12.5 $\mathrm{mg} \mathrm{g}^{-1}$ substrate). Samples were incubated in a $50 \mathrm{mM}$ ammonium acetate buffer ( $\mathrm{pH}$ 5.0) containing ascorbic acid addition (1 $\mathrm{mM}$ ) for $24 \mathrm{~h}$ at $52^{\circ} \mathrm{C}$. In all three incubations, MtLPMO9A released nonoxidized xylo-oligomers $\left(\mathrm{XOS}_{n}\right)$. A - incubation of WAX with MtLPMO9A; formation of non-oxidized xylo-oligomers and traces of acetylated (acety $X$ $\mathrm{OS}_{\mathrm{n}}$ ) xylo-oligomers (+42 Da). B - incubation of BiWX with MtLPMO9A; formation of non-oxidized xylo-oligomers and xylo-oligomers $\left(\mathrm{Gl}_{\mathrm{me}} \mathrm{XOS}_{\mathrm{n}}\right)$ substituted with 4-O-methyl-glucoronic acid (+191 Da). C - incubation of OSX with MtLPMO9A releases non-oxidized xylo-oligomers only. Masses represents lithium (Li) adducts only.

Additional file 4: Figure 4. The HPAEC elution patterns of xylan incubated with MtLPMO9A. Birchwood xylan (BiWX), oat spelt xylan (OSX) and wheat arabinoxylan (WAX) $\left(2 \mathrm{mg} \mathrm{mL}^{-1}\right)$ before and after incubation with MtLPMO9A (12.5 mg g $\mathrm{g}^{-1}$ substrate). Samples were incubated in a $50 \mathrm{mM}$ ammonium acetate buffer ( $\mathrm{pH}$ 5.0) containing ascorbic acid addition (1 $\mathrm{mM}$ ) for $24 \mathrm{~h}$ at $52^{\circ} \mathrm{C}$. Incubation with MtLPMO9A results in the formation of non-oxidized linear xylo-oligomers $\left(\mathrm{XOS}_{n}\right)$ and substituted xylo-oligomers (black dashed arrow).

Additional file 5: Figure 5. The HPAEC elution patterns of MtLPMO9A incubations with xylan and xylan-RAC mixtures. (A) birchwood xylan (BiWX), (B) oat spelt xylan (OSX) and (C) wheat arabinoxylan (WAX) (2 mg $\left.\mathrm{mL}^{-1}\right)$ in the presence and absence of regenerated amorphous cellulose (RAC; $2 \mathrm{mg} \mathrm{mL}^{-1}$ ) before and after incubation with MtLPMO9A (12.5 $\mathrm{mg} \mathrm{g}^{-1}$ substrate). Samples were incubated in a $50 \mathrm{mM}$ ammonium acetate buffer (pH 5.0) with ascorbic acid addition ( $1 \mathrm{mM}$ ) or without for $24 \mathrm{~h}$ at $52^{\circ} \mathrm{C}$. Incubation with MtLPMO9A of all three xylans and xylan-RAC mixtures, in the presence or absence of ascorbic acid, results in the formation of non-oxidized linear xylo-oligomers ( $\mathrm{XOS}_{n}$ ) and substituted xylo-oligomers (black dashed arrow). Incubation of xylan-RAC mixtures with MtLPMO9A in the presence of ascorbic acid results in the formation of non-oxidized gluco-oligomers (GlcOS $)$ and C1-oxidized gluco-oligomers (GlcOS $B$ - The incubation of MtLPMO9A with BiWX-RAC and OSX-RAC mixture in the presence of ascorbic acid results in the formation of numerous products (black arrow), which are not present if MtLPMO9A is incubated with BiWX, OSX or RAC alone. The results of MALDI-TOF MS analysis of BiWX-RAC and OSX-RAC mixture incubated with MtLPMO9A in the presence of ascorbic acid are shown in Figure 4. 
Additional file 6: Figure 6. The HPAEC and MALDI-TOF MS analysis of oat spelt (OS) $\beta$-glucan incubated with MtLPMO9A. A - HPAEC elution pattern OS $\beta$-glucan before and after incubation with partially purified MtLPMO9A fraction SEC-I (Additional Figure 1; $12.5 \mathrm{mg} \mathrm{g}^{-1}$ substrate), with addition of $1 \mathrm{mM}$ ascorbic acid or without. A - In the presence and absence of ascorbic acid, various non-oxidized $\beta$-gluco-oligomers $\left(G_{c O S}\right)$ are formed by the partially purified MtLPMO9A fraction. Incubation of oat spelt $\beta$-glucan with MtLPMO9A in the presence of ascorbic acid results in the formation of oxidized gluco-oligomers $\left(\mathrm{GlCOS}_{n^{\prime}}^{\#} \mathrm{GlCOS}_{n}{ }^{*}\right)$. B - MALDI-TOF mass spectrum of partially purified MtLPMO9A incubated with oat spelt $\beta$-glucan in the presence of $1 \mathrm{mM}$ ascorbic acid. Clusters of non-oxidized gluco-oligomers and gluco-oligomers, oxidized at C1 $\left(\mathrm{GlCOS}_{n}^{\#)}\right.$ and $\mathrm{C} 4\left(\mathrm{GlcOS}_{n}^{*}\right)$ are determined. Enlargement (C) shows the presence of non-oxidized gluco-oligomers and gluco-oligomers, oxidized at $\mathrm{C} 1$ with an aldonic acid $\left({ }^{*}\right)$ and at $\mathrm{C} 4$ with a keto-group $\left({ }^{*}\right)$. Masses represent lithium (Li) adducts only.

Additional file 7: Figure 7. The HPAEC and MALDI-TOF MS analysis of xyloglucan incubated with MtLPMO9A. A - HPAEC elution pattern of xyloglucan from tamarind seed $\left(2 \mathrm{mg} \mathrm{mL}^{-1}\right)$ after incubation with partially purified MtLPMO9A fraction SEC-I (Additional Figure $1 ; 12.5 \mathrm{mg} \mathrm{g}^{-1}$ substrate). Samples were incubated in $50 \mathrm{mM}$ ammonium acetate buffer (pH $5.0)$ for $24 \mathrm{~h}$ at $52^{\circ} \mathrm{C}$, either with ascorbic acid addition ( $1 \mathrm{mM}$ ) or without. Numerous various non-oxidized xyloglucan-derived oligomers were formed if xyloglucan was incubated with MtLPMO9A, either with ascorbic acid addition (1 mM) or without. B - MALDI-TOF mass spectrum of xyloglucan incubated with MtLPMO9A with $1 \mathrm{mM}$ ascorbic acid addition. Clusters of non-oxidized and oxidized xyloglucan-derived oligomers are formed. C (enlargement of B) - In the presence of MtLPMO9A and ascorbic acid, next to non-oxidized xyloglucan-derived oligomers, oligomers oxidized at the $\mathrm{C} 1\left(^{*}\right)$ and $C 4\left(^{*}\right)$ position are formed. Masses represent lithium (Li) adducts only.

Additional file 8: Figure 8. MtLPMO9A incubation with a cellulase cocktail. HPAEC elution patterns of Avicel incubations with a cellulase cocktail (Dyadic, Wageningen, The Nederlands) with and without partially purified MtLPMO9A addition (2.5 $\mathrm{mg}$ protein $\mathrm{g}^{-1}$ Avicel). The addition of MtLPMO9A to a cellulase cocktail (5 $\mathrm{mg}$ protein $\mathrm{g}^{-1}$ Avicel) results in a $60 \%$ higher release of glucose (based on HPAEC-area) compared to the glucose release from Avicel by the cellulase cocktail alone. Samples were incubated in $50 \mathrm{mM}$ acetate buffer $\left(\mathrm{pH} \mathrm{5.0)}\right.$ ) at $52^{\circ} \mathrm{C}$ with ascorbic acid addition (1 $\mathrm{mM})$.

\section{Abbreviations}

AA: auxiliary activity; BiWX: birchwood glucuronoxylan; CAZy: carbohydrateactive enzymes; EGI: endoglucanase l; GH: glycoside hydrolases; GIcAmeXOS: 4-O-methylglucoronic acid containing C1-oxidized xylo-oligosaccharides; GIcAmeXOS *: 4-O-methylglucoronic acid containing C4-oxidized xylooligosaccharides; GlcAmeXOS : 4-O-methylglucoronic acid containing nonoxidized xylo-oligosaccharides; GlcOS ${ }_{n}^{\#:}$ C1-oxidized gluco-oligosaccharides; $\mathrm{GlCOS}_{n}{ }^{*}$ : C4 oxidized gluco-oligosaccharides; GlcOS : non-oxidized glucooligosaccharides; HPAEC: high performance anion exchange chromatography; LC/ESI-MS: liquid chromatography/electronspray ionization-mass spectrometry; LPMO: Iytic polysaccharide monooxygenases; MALDI-TOF MS: matrix-assisted laser desorption ionization-time of flight mass spectrometry; OSX: oat spelt xylan; PAD: pulsed amperometric detection; RAC: regenerated amorphous cellulose; SDS-PAGE: sodium dodecyl sulfate-polyacrylamide gel electrophoresis; TIC: total ion current; WAX: wheat arabinoxylan; $\mathrm{XOS}_{n}^{\#}$ : C1-oxidized xylo-oligosaccharides; XOS $_{n}^{*}$ : C4-oxidized xylo-oligosaccharides; $\mathrm{XOS}_{n}$ : non-oxidized xylo-oligosaccharides.

\section{Authors' contributions}

All authors intellectually contributed to this study. MF, JV, SWAH, MJK, WJHB, $\mathrm{HG}$ and MAK made substantial contribution to the conception and design. MF, SS and AHW carried out the experiments and data analysis. MF prepared the manuscript. All authors were involved in drafting the manuscript or revising it critically for the intellectual content. All authors read and approved the final manuscript.

\section{Author details}

${ }^{1}$ Laboratory of Food Chemistry, Wageningen University, Bornse Weilanden 9, 6708 WG Wageningen, The Netherlands. ${ }^{2}$ Department of Food Science, University of Parma, Parco Area delle Scienze 59a, University Campus, 43124 Parma, Italy. ${ }^{3}$ Laboratory of Biochemistry, Wageningen University, Wageningen, The Netherlands. ${ }^{4}$ Dyadic Netherlands, Nieuwe Kanaal 7-S, 6709 PA Wageningen, The Netherlands.

\section{Acknowledgements}

This research was funded by the VLAG Graduate School (Advanced Studies in Food Technology, Agrobiotechnology, Nutrition and Health Sciences) of Wageningen University (Wageningen, The Netherlands).

\section{Compliance with ethical guidelines}

\section{Competing interests}

The authors declare that they have no competing interests.

Received: 7 April 2015 Accepted: 8 July 2015

Published online: 17 July 2015

\section{References}

1. Hinz SWA, Pouvreau L, Joosten R, Bartels J, Jonathan MC, Wery J et al (2009) Hemicellulase production in Chrysosporium lucknowense C1. J Cereal Sci 50(3):318-323. doi:10.1016/j.jcs.2009.07.005

2. Vincken JP, de Keizer A, Beldman G, Voragen AGJ (1995) Fractionation of xyloglucan fragments and their interaction with cellulose. Plant Physiol 108(4):1579-1585. doi:10.1104/pp.108.4.1579

3. Lam TBT, Kadoya K, liyama K (2001) Bonding of hydroxycinnamic acids to lignin: ferulic and $p$-coumaric acids are predominantly linked at the benzyl position of lignin, not the $\beta$-position, in grass cell walls. Phytochemistry 57(6):987-992. doi:10.1016/S0031-9422(01)00052-8

4. Kabel MA, Van den Borne H, Vincken JP, Voragen AGJ, Schols HA (2007) Structural differences of xylans affect their interaction with cellulose. Carbohydr Polym 69(1):94-105. doi:10.1016/j.carbpol.2006.09.006

5. Carpita NC, Gibeaut DM (1993) Structural models of primary cell walls in flowering plants: consistency of molecular structure with the physical properties of the walls during growth. Plant J 3(1):1-30. doi:10.1111/ j.1365-313X.1993.tb00007.x

6. Zhang YHP, Lynd LR (2004) Toward an aggregated understanding of enzymatic hydrolysis of cellulose: noncomplexed cellulase systems. Biotechnol Bioeng 88(7):797-824. doi:10.1002/bit.20282

7. Parthasarathi R, Bellesia G, Chundawat SPS, Dale BE, Langan P, Gnanakaran S (2011) Insights into hydrogen bonding and stacking interactions in cellulose. J Phys Chem A. 115(49):14191-14202. doi:10.1021/jp203620x

8. Mansfield SD, Mooney C, Saddler JN (1999) Substrate and enzyme characteristics that limit cellulose hydrolysis. Biotechnol Prog 15(5):804-816. doi:10.1021/bp9900864

9. Jacquet G, Pollet B, Lapierre C, Mhamdi F, Rolando C (1995) New ether linked ferulic acid conferyl alcohol dimers identified in grass straws. J Agric Food Chem 43(10):2746-2751. doi:10.1021/jf00058a037

10. Takahashi N, Koshijima T (1988) Ester linkages between lignin and glucuronoxylan in a lignin-carbohydrate complex from beech (Fagus crenata) wood. Wood Sci Technol 22(231-241):231-241. doi:10.1007/BF00386018

11. Hoffman M, Jia Z, Peña MJ, Cash M, Harper A, Blackburn li AR et al (2005) Structural analysis of xyloglucans in the primary cell walls of plants in the subclass Asteridae. Carbohydr Res 340(11):1826-1840. doi:10.1016/j. carres.2005.04.016

12. Popper ZA, Fry SC (2003) Primary cell wall composition of Bryophytes and Charophytes. Ann Bot 91(1):1-12. doi:10.1093/aob/mcg013

13. Bochicchio R, Reicher F (2003) Are hemicelluloses from Podocarpus lambertii typical of gymnosperms? Carbohydr Polym 53(2):127-136. doi:10.1016/S0144-8617(03)00046-8

14. Capek P, Kubačková M, Alföldi J, Bilisics L, Lišková D, Kákoniová D (2000) Galactoglucomannan from the secondary cell wall of Picea abies L. Karst. Carbohydr Res 329(3):635-645. doi:10.1016/S0008-6215(00)00210-X 
15. Vogel J (2008) Unique aspects of the grass cell wall. Curr Opin Plant Biol 11(3):301-307. doi:10.1016/j.pbi.2008.03.002

16. Smith BG, Harris PJ (1999) The polysaccharide composition of Poales cell walls: Poaceae cell walls are not unique. Biochem Syst Ecol 27(1):33-53. doi:10.1016/S0305-1978(98)00068-4

17. Ebringerová A, Hromádková Z, Heinze T (2005) Hemicellulose. Adv Polym Sci 186:1-67. doi:10.1007/b136816

18. Yang B, Wyman CE (2004) Effect of xylan and lignin removal by batch and flowthrough pretreatment on the enzymatic digestibility of corn stover cellulose. Biotechnol Bioeng 86(1):88-95. doi:10.1002/bit.20043

19. Jeoh T, Ishizawa Cl, Davis MF, Himmel ME, Adney WS, Johnson DK (2007) Cellulase digestibility of pretreated biomass is limited by cellulose accessibility. Biotechnol Bioeng 98(1):112-122. doi:10.1002/bit.21408

20. CAZy (2014) Glycoside Hydrolase family classification. doi:www.cazy.org/ Glycoside-Hydrolases.html. Accessed 27 Mar 2015

21. Levasseur A, Drula E, Lombard V, Coutinho PM, Henrissat B (2013) Expansion of the enzymatic repertoire of the CAZy database to integrate auxiliary redox enzymes. Biotechnol Biofuels 6:41. doi:10.1186/1754-6834-6-41

22. Vu W, Beeson WT, Phillips CM, Cate JH, Marletta MA (2014) Determinants of regioselective hydroxylation in the fungal polysaccharide monooxygenases. J Am Chem Soc 136(2):562-565. doi:10.1021/ja409384b

23. Isaksen T, Westereng B, Aachmann FL, Agger JW, Kracher D, Kittl R et al (2014) A C4-oxidizing lytic polysaccharide monooxygenase cleaving both cellulose and cello-oligosaccharides. J Biol Chem 289(5):2632-2642. doi:10.1074/jbc.M113.530196

24. Agger JW, Isaksen T, Varnai A, Vidal-Melgosa S, Willats WG, Ludwig R et al (2014) Discovery of LPMO activity on hemicelluloses shows the importance of oxidative processes in plant cell wall degradation. Proc Natl Acad Sci USA 111(17):6287-6292. doi:10.1073/pnas.1323629111

25. Forsberg Z, Rohr AK, Mekasha S, Andersson KK, Eijsink VG, Vaaje-Kolstad G et al (2014) Comparative study of two chitin-active and two celluloseactive AA10-type lytic polysaccharide monooxygenases. Biochemistry 53(10):1647-1656. doi:10.1021/bi5000433

26. Lo Leggio L, Simmons TJ, Poulsen J-CN, Frandsen KEH, Hemsworth GR, Stringer MA et al (2015) Structure and boosting activity of a starch-degrading lytic polysaccharide monooxygenase. Nat Commun. doi:10.1038/ncomms6961

27. Vu W, Beeson WT, Span EA, Farquhar ER, Marletta MA (2014) A family of starch-active polysaccharide monooxygenases. Proc Natl Acad Sci USA 111(38):13822-13827. doi:10.1073/pnas.1408090111

28. Westereng B, Ishida T, Vaaje-Kolstad G, Wu M, Eijsink VG, Igarashi K et al (2011) The putative endoglucanase PCGH61D from Phanerochaete chrysosporium is a metal-dependent oxidative enzyme that cleaves cellulose. PLoS One 6(11):e27807. doi:10.1371/journal.pone.0027807

29. Harris PV, Welner D, McFarland KC, Re E, Navarro Poulsen JC, Brown K et al (2010) Stimulation of lignocellulosic biomass hydrolysis by proteins of glycoside hydrolase family 61: structure and function of a large, enigmatic family. Biochemistry 49(15):3305-3316. doi:10.1021/bi100009p

30. Hemsworth GR, Davies GJ, Walton PH (2013) Recent insights into coppercontaining lytic polysaccharide mono-oxygenases. Curr Opin Struct Biol 23(5):660-668. doi:10.1016/j.sbi.2013.05.006

31. Phillips CM, Beeson WT, Cate JH, Marletta MA (2011) Cellobiose dehydrogenase and a copper-dependent polysaccharide monooxygenase potentiate cellulose degradation by Neurospora crassa. ACS Chem Biol 6(12):1399-1406. doi:10.1021/cb200351y
32. Beeson WT, Phillips CM, Cate JH, Marletta MA (2012) Oxidative cleavage of cellulose by fungal copper-dependent polysaccharide monooxygenases. J Am Chem Soc 134(2):890-892. doi:10.1021/ja210657t

33. Kim S, Stahlberg J, Sandgren M, Paton RS, Beckham GT (2014) Quantum mechanical calculations suggest that lytic polysaccharide monooxygenases use a copper-oxyl, oxygen-rebound mechanism. Proc Natl Acad Sci USA 111(1):149-154. doi:10.1073/pnas.1316609111

34. Guillotin SE, Van Kampen J, Boulenguer P, Schols HA, Voragen AGJ (2006) Degree of blockiness of amide groups as indicator for difference in physical behavior of amidated pectins. Biopolymers 82(1):29-37. doi:10.1002/ bip.20456

35. Van Gool MP (2012) Targeted discovery and functional characterisation of complex-xylan degrading enzymes. PhD Thesis. Wageningen University, Wageningen

36. Beldman G, Voragen AGJ, Rombouts FM, Searle-van Leeuwen MF, Pilnik W (1987) Adsorption and kinetic behavior of purified endoglucanases and exoglucanases from Trichoderma viride. Biotechnol Bioeng 30(2):251-257. doi:10.1002/bit.260300215

37. Vincken J-P, Beldman G, Voragen AGJ (1997) Substrate specificity of endoglucanases: what determines xyloglucanase activity? Carbohydr Res 298(4):299-310. doi:10.1016/S0008-6215(96)00325-4

38. Van Gool MP, van Muiswinkel GC, Hinz SW, Schols HA, Sinitsyn AP, Gruppen H (2012) Two GH10 endo-xylanases from Myceliophthora thermophila C1 with and without cellulose binding module act differently towards soluble and insoluble xylans. Bioresour Technol 119:123-132. doi:10.1016/j.biortech.2012.05.117

39. Van Gool MP, Vancsó I, Schols HA, Toth K, Szakacs G, Gruppen H (2011) Screening for distinct xylan degrading enzymes in complex shake flask fermentation supernatants. Bioresour Technol 102(10):6039-6047. doi:10.1016/j.biortech.2011.02.105

40. Visser H, Joosten V, Punt PJ, Gusakov AV, Olson PT, Joosten R et al (2011) Development of a mature fungal technology and production platform for industrial enzymes based on a Myceliophthora thermophila isolate, previously known as Chrysosporium lucknowense C1. Ind Biotechnol 7:214-223. doi:10.1089/ind.2011.0003

41. Punt PJ, Burlingame RP, Pynnonen CM, Olson PT, Wery J, Visser J, Heinrich et al (2010) Chrysosporium lucknowense protein production system. Patent WO/2010/107303

42. Zhang YHP, Cui J, Lynd LR, Kuang LR (2006) A transition from cellulose swelling to cellulose dissolution by o-phosphoric acid: evidence from enzymatic hydrolysis and supramolecular structure. Biomacromolecules 7(2):644-648. doi:10.1021/bm050799c

43. Sali A (1995) Comparative protein modeling by satisfaction of spatial restraints. Mol Med Today 1(6):270-277. doi:10.1016/ S1357-4310(95)91170-7

44. Eswar N, Webb B, Marti-Renom MA, Madhusudhan MS, Eramian D, Shen MY et al (2007) Comparative protein structure modeling using MODELLER. Curr Protocols Protein Sci. doi:10.1002/0471140864.ps0209s50

\section{Submit your next manuscript to BioMed Central and take full advantage of:}

- Convenient online submission

- Thorough peer review

- No space constraints or color figure charges

- Immediate publication on acceptance

- Inclusion in PubMed, CAS, Scopus and Google Scholar

- Research which is freely available for redistribution

Submit your manuscript at

www.biomedcentral.com/submit
C Biomed Central 